\title{
The discrete dynamics of monotonically decomposable maps
}

\author{
H. L. Smith
}

Published online: 24 January 2008

(C) Springer-Verlag 2008

Erratum to: J. Math. Biol. (2006) 53: 747-758

DOI 10.1007/s00285-006-0004-3

\begin{abstract}
We correct errors in Propositions 11 and 14 of the paper in the title. Corollary 13 is withdrawn.
\end{abstract}

\section{Introduction}

The purpose of this communication is to point out an error in Propositions 11 and 14 of [1]. Both results require an additional hypothesis to be described below. The author would like to thank Patrick de Leenheer for pointing out the error. Unfortunately, the author is unable to verify the additional hypothesis of Proposition 11 in the application to the LPA model, Corollary 13. Therefore Corollary 13 is withdrawn.

The error consists of an incorrect attempt to verify hypothesis (7) of Corollary 3 in the proof of Proposition 11; similarly, hypothesis (iii) of Theorem 9 is problematic in Proposition 14. It does not seem possible to fix this argument in general. Therefore, the additional hypothesis

$$
e \leq a \leq b, \quad A(b) a+e=a, \quad A(a) b+e=b \Rightarrow a=b
$$

The online version of the original article can be found under doi:10.1007/s00285-006-0004-3.

H. L. Smith $(\varangle)$

Department of Mathematics and Statistics,

Arizona State University, Tempe, AZ 85287, USA

e-mail: halsmith@asu.edu 
to Propositions 11 and 14 appear to be required. Assuming (1), both results hold since (1) ensures that hypothesis (7) of Corollary 3 and hypothesis (iii) of Theorem 9 hold. Unfortunately, (1) is difficult to verify. It requires showing that $F(x)=(I-A(x))^{-1} e$ has no ordered period two points.

\section{Reference}

1. Smith, H.L.: The discrete dynamics of monotonically decomposable maps. J. Math. Biol. 53, 747758 (2006) 